\title{
A Bayesian Super-Resolution Approach to Demosaicing of Blurred Images
}

\author{
Miguel Vega, ${ }^{1}$ Rafael Molina, ${ }^{2}$ and Aggelos K. Katsaggelos ${ }^{3}$ \\ ${ }^{1}$ Departamento de Lenguajes y Sistemas Informáticos, Escuela Técnica Superior de Ingeniería Infomática, Universidad de Granada, \\ 18071 Granada, Spain \\ ${ }^{2}$ Departamento de Ciencias de la Computación e Inteligencia Artificial, Escuela Técnica Superior de Ingeniería Infomática, \\ Universidad de Granada, 18071 Granada, Spain \\ ${ }^{3}$ Department of Electrical Engineering and Computer Science, Robert R. McCormick School of Engineering and Applied Science, \\ Northwestern University, Evanston, IL 60208-3118, USA
}

Received 10 December 2004; Revised 6 May 2005; Accepted 18 May 2005

\begin{abstract}
Most of the available digital color cameras use a single image sensor with a color filter array (CFA) in acquiring an image. In order to produce a visible color image, a demosaicing process must be applied, which produces undesirable artifacts. An additional problem appears when the observed color image is also blurred. This paper addresses the problem of deconvolving color images observed with a single coupled charged device (CCD) from the super-resolution point of view. Utilizing the Bayesian paradigm, an estimate of the reconstructed image and the model parameters is generated. The proposed method is tested on real images.
\end{abstract}

Copyright (c) 2006 Hindawi Publishing Corporation. All rights reserved.

\section{INTRODUCTION}

Most digital color cameras use a single coupled charge device (CCD), or a single CMOS sensor, with a color filter array (CFA) to acquire color images. Unfortunately, the color filter generates different spectral responses at every CCD cell. The most widely used CFA is the Bayer one [1]. It imposes a spatial pattern of two G cells, one R, and one B cell, as shown in Figure 1.

Bayer camera pixels convey incomplete color information which needs to be extended to produce a visible color image. Such color processing is known as demosaicing (or demosaicking). From the pioneering work of Bayer [1] to nowadays, a lot of work has been devoted to the demosaicing topic (see [2] for a review). The use of a CFA and the corresponding demosaicing process produce undesirable artifacts, which are difficult to avoid. Among such artifacts are the zipper effect, also known as color fringe, and the appearance of moiré patterns.

Different interpolation techniques have been applied to demosaicing. Cok [3] applied bilinear interpolation to the G channel first, since it is the most populated and is supposed to apport information about luminance, and then applied bilinear interpolation to the chrominance ratios $\mathrm{R} / \mathrm{G}$ and $\mathrm{B} / \mathrm{G}$. Freeman [4] applied a median filter to the differences between bilineraly interpolated values of the different channels, and based on these and the observed channel at every pixel, the intensities of the two other channels are estimated. An improvement of this technique was to perform adaptive interpolation considering chrominance gradients, so as to take into account edges between objects [5]. This technique was further improved in [6] where steerable inverse diffusion in color was also applied. In [7], interchannel correlations were considered in an alternating-projections scheme. Finally in [8], a new orthogonal wavelet representation of multivalued images was applied. No much work has been reported on the problem of deconvolving single-CCD observed color images.

Over the last two decades, research has been devoted to the problem of reconstructing a high-resolution image from multiple undersampled, shifted, degraded frames with subpixel displacement errors (see, e.g., [9-17]). Super-resolution has only been applied recently to demosaicing problems [1821]. Unfortunately, again, few results (see [19-21]) have been reported on the deconvolution of such images. In our previous work [22, 23], we addressed the high-resolution problem from complete and also from incomplete observations within the general framework of frequency-domain multichannel signal processing developed in [24]. In this paper, we formulate the demosaicing problem as a high-resolution problem from incomplete observations, and therefore we propose a new way to look at the problem of deconvolution.

The rest of the paper is organized as follows. The problem formulation is described in Section 2. In Section 3, we describe the model used to reconstruct each band of the color 


\begin{tabular}{|c|c|c|c|c|c|c|c|}
\hline G & $\mathrm{R}$ & $\mathrm{G}$ & $\mathrm{R}$ & $\mathrm{G}$ & $\mathrm{R}$ & $\mathrm{G}$ & $\mathrm{R}$ \\
\hline B & G & B & G & B & G & B & $\mathrm{G}$ \\
\hline G & $\mathrm{R}$ & $\mathrm{G}$ & $\mathrm{R}$ & $\mathrm{G}$ & $\mathrm{R}$ & $\mathrm{G}$ & $\mathrm{R}$ \\
\hline B & $\mathrm{G}$ & B & $\mathrm{G}$ & B & $\mathrm{G}$ & B & $\mathrm{G}$ \\
\hline G & $\mathrm{R}$ & G & $\mathrm{R}$ & $\mathrm{G}$ & $\mathrm{R}$ & $\mathrm{G}$ & $\mathrm{R}$ \\
\hline B & G & B & $\mathrm{G}$ & B & $\mathrm{G}$ & B & $\mathrm{G}$ \\
\hline G & $\mathrm{R}$ & $\mathrm{G}$ & $\mathrm{R}$ & $\mathrm{G}$ & $\mathrm{R}$ & $\mathrm{G}$ & $\mathrm{R}$ \\
\hline B & G & B & $\mathrm{G}$ & B & $\mathrm{G}$ & B & G \\
\hline
\end{tabular}

(a)

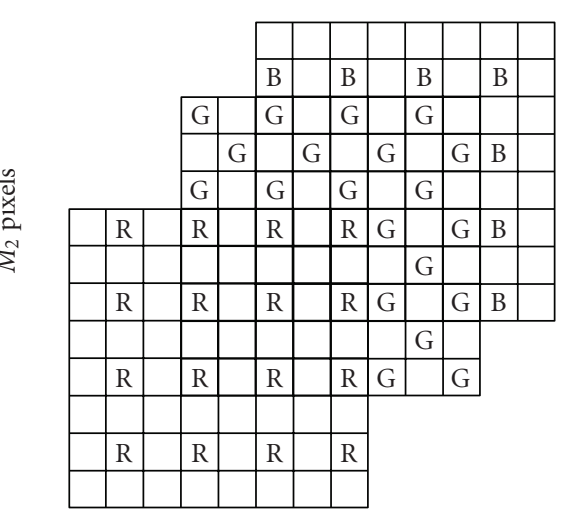

(b)

Figure 1: (a) Pattern of channel observations for a Bayer camera with CFA; (b) observed low-resolution channels (the array in (a) and all the arrays in (b) are of the same size).

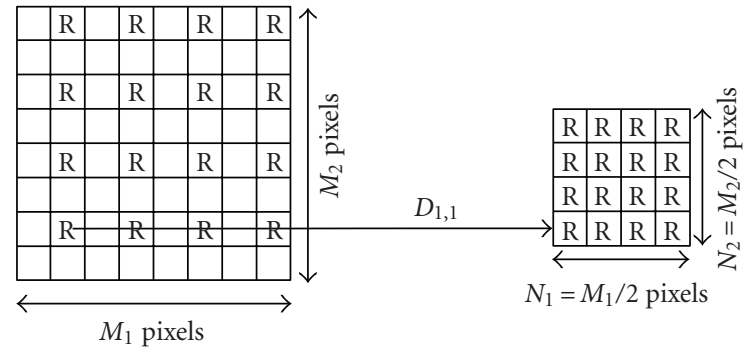

FIgURE 2: Process to obtain the low-resolution observed R channel.

image and then examine how to iteratively estimate the highresolution color image. The consistency of the global distribution on the color image is studied in Section 4. Experimental results are described in Section 5. Finally, Section 6 concludes the paper.

\section{PROBLEM FORMULATION}

Consider a Bayer camera with a color filter array (CFA) over one CCD with $M_{1} \times M_{2}$ pixels, as shown in Figure 1(a). Assuming that the camera has three $M_{1} \times M_{2}$ CCDs, one for each of the R, G, B channels, the observed image is given by

$$
\mathbf{g}=\left(\mathbf{g}^{R t}, \mathbf{g}^{G t}, \mathbf{g}^{B t}\right)^{t},
$$

where $t$ denotes the transpose of a vector or a matrix and each one of the $M_{1} \times M_{2}$ column vectors $\mathbf{g}^{c}, c \in\{\mathrm{R}, \mathrm{G}, \mathrm{B}\}$, results from the lexicographic ordering of the two-dimensional signal in the R, G, and B channels, respectively.

Due to the presence of the CFA, we do not observe $\mathrm{g}$ but an incomplete subset of it, see Figure 1(b). Let us characterize these observed values in the Bayer camera. Let $N_{1}=M_{1} / 2$ and $N_{2}=M_{2} / 2$; then the $1 \mathrm{D}$ downsampling matrices $\mathrm{D}_{l}^{x}$ and $\mathbf{D}_{l}^{y}$ are defined by

$$
\mathbf{D}_{l}^{x}=\mathbf{I}_{N_{1}} \otimes \mathbf{e}_{l}^{t}, \quad \mathbf{D}_{l}^{y}=\mathbf{I}_{N_{2}} \otimes \mathbf{e}_{l}^{t},
$$

where $\mathbf{I}_{N_{i}}$ is the $N_{i} \times N_{i}$ identity matrix, $\mathbf{e}_{l}$ is a $2 \times 1$ unit vector whose nonzero element is in the $l$ th position, $l \in\{0,1\}$, and $\otimes$ denotes the Kronecker product operator. The $\left(N_{1} \times N_{2}\right) \times$ $\left(M_{1} \times M_{2}\right) 2 \mathbf{D}$ downsampling matrix is now given by $\mathbf{D}_{l 1, l 2}=$ $\mathbf{D}_{l 1}^{x} \otimes \mathbf{D}_{l 2}^{y}$.

Using the above downsampling matrices, the subimage of $\mathbf{g}$ which has been observed, $\mathbf{g}^{\text {obs }}$, may be viewed as the incomplete set of $N_{1} \times N_{2}$ low-resolution images

$$
\mathbf{g}^{\text {obs }}=\left(\mathbf{g}_{1,1}^{R t}, \mathbf{g}_{1,0}^{G t}, \mathbf{g}_{0,1}^{G t}, \mathbf{g}_{0,0}^{B t}\right)^{t},
$$

where

$$
\begin{array}{ll}
\mathbf{g}_{1,1}^{\mathrm{R}}=\mathbf{D}_{1,1} \mathbf{g}^{\mathrm{R}}, & \mathbf{g}_{1,0}^{\mathrm{G}}=\mathbf{D}_{1,0} \mathbf{g}^{\mathrm{G}}, \\
\mathbf{g}_{0,1}^{\mathrm{G}}=\mathbf{D}_{0,1} \mathbf{g}^{\mathrm{G}}, & \mathbf{g}_{0,0}^{\mathrm{B}}=\mathbf{D}_{0,0} \mathbf{g}^{\mathrm{B}} .
\end{array}
$$

As an example, Figure 2 illustrates how $\mathbf{g}_{1,1}^{\mathrm{R}}$ is obtained. Note that the origin of coordinates is located in the bottomleft side of the array. We have one observed $N_{1} \times N_{2}$ lowresolution image at $\mathrm{R}$, two at $\mathrm{G}$, and one at $\mathrm{B}$ channels.

In order to deconvolve the observed image, the image formation process has to take into account the presence of blurring. We assume that $\mathbf{g}$ in (1) can be written as

$$
\mathbf{g}=\left(\begin{array}{l}
\mathbf{g}^{\mathrm{R}} \\
\mathbf{g}^{\mathrm{G}} \\
\mathbf{g}^{\mathrm{B}}
\end{array}\right)=\left(\begin{array}{l}
\mathbf{B} \mathbf{f}^{\mathrm{R}} \\
\mathbf{B} \mathbf{f}^{\mathrm{G}} \\
\mathbf{B f}^{\mathrm{B}}
\end{array}\right)+\left(\begin{array}{l}
\mathbf{n}^{\mathrm{R}} \\
\mathbf{n}^{\mathrm{G}} \\
\mathbf{n}^{\mathrm{B}}
\end{array}\right)=\left(\begin{array}{ccc}
\mathbf{B} & 0 & 0 \\
0 & \mathbf{B} & 0 \\
0 & 0 & \mathbf{B}
\end{array}\right) \mathbf{f}+\mathbf{n},
$$




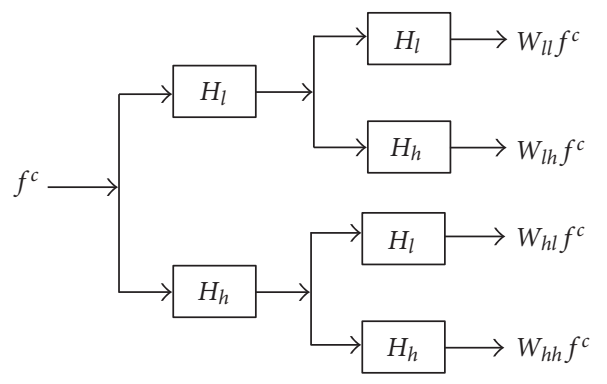

Figure 3: Two-level filter bank.

where $\mathbf{B}$ is an $\left(M_{1} \times M_{2}\right) \times\left(M_{1} \times M_{2}\right)$ matrix that defines the systematic blur of the camera, assumed to be known and approximated by a block circulant matrix, $\mathbf{f}$ denotes the real underlying high-resolution color image we are trying to estimate, and $\mathbf{n}$ denotes white independent uncorrelated noise between and within channels with variance $1 / \beta^{c}$ in channel $c \in\{R, G, B\}$. See [25] and references therein for a complete description of the blurring process in color images. Substituting this equation in (4), we have that the discrete lowresolution observed images can be written as

$$
\begin{array}{ll}
\mathbf{g}_{1,1}^{\mathrm{R}}=\mathbf{D}_{1,1} \mathbf{B} \mathbf{f}^{\mathrm{R}}+\mathbf{D}_{1,1} \mathbf{n}^{\mathrm{R}}, & \mathbf{g}_{1,0}^{\mathrm{G}}=\mathbf{D}_{1,0} \mathbf{B} \mathbf{f}^{\mathrm{G}}+\mathbf{D}_{1,0} \mathbf{n}^{\mathrm{G}}, \\
\mathbf{g}_{0,1}^{\mathrm{G}}=\mathbf{D}_{0,1} \mathbf{B} \mathbf{f}^{\mathrm{G}}+\mathbf{D}_{0,1} \mathbf{n}^{\mathrm{G}}, & \mathbf{g}_{0,0}^{\mathrm{B}}=\mathbf{D}_{0,0} \mathbf{B} \mathbf{f}^{\mathrm{B}}+\mathbf{D}_{0,0} \mathbf{n}^{\mathrm{R}},
\end{array}
$$

where we have the following distributions for the subsampled noise:

$$
\begin{array}{ll}
\mathbf{D}_{1,1} \mathbf{n}^{\mathrm{R}} \sim N\left(0,\left(1 / \beta^{\mathrm{R}} I_{N_{1} \times N_{2}}\right)\right), & \mathbf{D}_{1,0} \mathbf{n}^{\mathrm{G}} \sim N\left(0,\left(1 / \beta^{\mathrm{G}} I_{N_{1} \times N_{2}}\right)\right), \\
\mathbf{D}_{0,1} \mathbf{n}^{\mathrm{G}} \sim N\left(0,\left(1 / \beta^{\mathrm{G}} I_{N_{1} \times N_{2}}\right)\right), & \mathbf{D}_{0,0} \mathbf{n}^{\mathrm{B}} \sim N\left(0,\left(1 / \beta^{\mathrm{B}} I_{N_{1} \times N_{2}}\right)\right) .
\end{array}
$$

From the above formulation, our goal has become the reconstruction of a complete RGB $M_{1} \times M_{2}$ high-resolution image $\mathbf{f}$ from the incomplete set of observations, $\mathbf{g}^{\text {obs }}$ in (3). In other words, our deconvolution problem has taken the form of a super-resolution reconstruction one. We can therefore apply the theory developed in $[23,26]$, by taking into account that we are dealing with multichannel images, and therefore the relationship between channels has to be included in the deconvolution process [25].

\section{BAYESIAN RECONSTRUCTION OF THE COLOR IMAGE}

Let us consider first the reconstruction of channel $c$ assuming that the observed data $\mathbf{g}^{\text {obs } c}$ and also the real images $\mathbf{f}^{c^{\prime}}$ and $\mathbf{f}^{c^{\prime \prime}}$, with $c^{\prime} \neq c$ and $c^{\prime \prime} \neq c$, are available.

In order to apply the Bayesian paradigm to this problem, we define $\mathrm{p}_{c}\left(\mathbf{f}^{c}\right), \mathrm{p}_{c}\left(\mathbf{f}^{\prime} \mid \mathbf{f}^{c}\right), \mathrm{p}_{c}\left(\mathbf{f}^{c^{\prime \prime}} \mid \mathbf{f}^{c}\right)$, and $\mathrm{p}_{c}\left(\mathbf{g}^{\text {obs }} \mid \mathbf{f}^{c}\right)$ and use the global distribution

$$
\begin{aligned}
\mathrm{p}_{c}\left(\mathbf{f}^{c}, \mathbf{f}^{c^{\prime}}, \mathbf{f}^{c^{\prime \prime}}, \mathbf{g}^{\text {obs } c}\right) \\
\quad=\mathrm{p}_{c}\left(\mathbf{f}^{c}\right) \mathrm{p}_{c}\left(\mathbf{f}^{c^{\prime}} \mid \mathbf{f}^{c}\right) \mathrm{p}_{c}\left(\mathbf{f}^{c^{\prime \prime}} \mid \mathbf{f}^{c}\right) \mathrm{p}_{c}\left(\mathbf{g}^{\text {obs } c} \mid \mathbf{f}^{c}\right) .
\end{aligned}
$$

Smoothness within channel $c$ is modelled by the introduction of the following prior distribution for $\mathbf{f}^{c}$ :

$$
\mathrm{p}\left(\mathbf{f}^{c}\left|\alpha^{c}\right|\right) \propto\left(\alpha^{c}\right)^{M_{1} \times M_{2} / 2} \exp \left[-\frac{1}{2} \alpha^{c}\left\|\mathbf{C} \mathbf{f}^{c}\right\|^{2}\right],
$$

where $\alpha^{c}>0$ and $\mathbf{C}$ denotes the Laplacian operator.

To define $\mathrm{p}_{c}\left(\mathbf{f}^{c^{\prime}} \mid \mathbf{f}^{c}\right)$ and similarly $\mathrm{p}_{c}\left(\mathbf{f}^{c^{\prime \prime}} \mid \mathbf{f}^{c}\right)$, we proceed as follows. A two-level bank of undecimated separable twodimensional filters constructed from a lowpass filter $H_{l}$ (with impulse response $\left.h_{l}=\left[\begin{array}{lll}1 & 2 & 1\end{array}\right] / 4\right)$ and a highpass filter $H_{h}$ $\left(h_{h}=\left[\begin{array}{ll}1-2 & 1\end{array}\right] / 4\right)$ is applied to $\mathbf{f}^{c^{\prime}}-\mathbf{f}^{c}$ obtaining the approximation subband $W_{l l}\left(\mathbf{f}^{c^{\prime}}-\mathbf{f}^{c}\right)$, and the horizontal $W_{l h}\left(\mathbf{f}^{c^{\prime}}-\mathbf{f}^{c}\right)$, vertical $W_{h l}\left(\mathbf{f}^{c^{\prime}}-\mathbf{f}^{c}\right)$, and diagonal $W_{h h}\left(\mathbf{f}^{c^{\prime}}-\mathbf{f}^{c}\right)$ detail subbands [7] (see Figure 3), where

$$
W_{u v}=H_{u} \otimes H_{v}, \quad \text { for } u v \in\{l l, l h, h l, h h\} .
$$

With these decomposition differences between channels, for high-frequency components are penalized by the introduction of the following probability distribution:

$$
\begin{aligned}
& \mathrm{p}_{c}\left(\mathbf{f}^{c^{\prime}} \mid \mathbf{f}^{c}, \gamma^{c c^{\prime}}\right) \propto\left|A\left(\gamma^{c c^{\prime}}\right)\right|^{-1 / 2} \\
& \quad \times \exp \left[-\frac{1}{2} \sum_{u v \in \mathscr{H} B} \gamma_{u v}^{c c^{\prime}} \|\left. W_{u v}\left(\mathbf{f}^{c^{\prime}}-\mathbf{f}^{c}\right)\right|^{2}\right],
\end{aligned}
$$

where $\mathscr{H} B=\{l h, h l, h h\}, \gamma_{u v}^{c c^{\prime}}$ measures the similarity of the $u v$ band of the $c$ and $c^{\prime}$ channels, $\gamma^{c c^{\prime}}=\left\{\gamma_{u v}^{c c^{\prime}} \mid u v \in \mathscr{H} B\right\}$, and

$$
A\left(\gamma^{c c^{\prime}}\right)=\sum_{u v \in \mathscr{H} B} \gamma_{u v}^{c c^{\prime}} W_{u v}^{t} W_{u v} .
$$

Before proceeding with the description of the observation model used in our formulation, we provide a justification of the prior model introduced at this point. The model is based on prior results in the literature. It was observed, for example, in [7] that for natural color images, there is a high correlation between red, green, and blue channels and that this correlation is higher for the high-frequency subbands $(l h, h l, h h)$. The effect of CFA sampling on these subbands was also examined in [7], where it was shown that the highfrequency subbands of the red and blue channels, especially the $l h$ and $h l$ subbands, are the ones affected the most by the downsampling process. Based on these observations, constraint sets were defined, within the POCS framework, that forced the high-frequency components of the red and blue channels to be similar to the high-frequency components of the green channel.

We initially followed the results in [7] within the Bayesian framework for demosaicing by introducing a prior that forced red and blue high-frequency components to be similar to those of the green channel. Using this prior, the improvements of the red and blue channels were in most cases higher, however, than the improvement corresponding to the green channel. This led us to introduce a prior, see (8) and (11), that favors similarity between the high-frequency components of all the three channels. The relative weights of the similarities between different channels are modulated by the $\gamma_{u v}^{c c^{\prime}}$ parameters, which are determined automatically by the proposed method, as explained below. 
From the model in (6), we have

$$
\begin{aligned}
& \mathrm{p}_{c}\left(\mathbf{g}^{\mathrm{obs} c} \mid \mathbf{f}^{c}, \beta^{c}\right) \\
& \propto\left\{\begin{array}{cc}
\beta^{\mathrm{R}^{N_{1} \times N_{2} / 2}} \exp \left[-\frac{\beta^{\mathrm{R}}}{2}\left\|\mathbf{g}_{1,1}^{\mathrm{R}}-\mathbf{D}_{1,1} \mathbf{B} \mathbf{f}^{\mathrm{R}}\right\|^{2}\right] & \text { if } c=\mathrm{R}, \\
\beta^{\mathrm{G}^{N_{1} \times N_{2}} \exp \left[-\frac{\beta^{\mathrm{G}}}{2}\left(\left\|\mathbf{g}_{1,0}^{\mathrm{G}}-\mathbf{D}_{1,0} \mathbf{B} \mathbf{f}^{\mathrm{G}}\right\|^{2}\right.\right.} \\
\left.\left.+\left\|\mathbf{g}_{0,1}^{\mathrm{G}}-\mathbf{D}_{0,1} \mathbf{B} \mathbf{f}^{\mathrm{G}}\right\|^{2}\right)\right] & \text { if } c=\mathrm{G}, \\
\beta^{\mathrm{B} N_{1} \times N_{2} / 2} \exp \left[-\frac{\beta^{\mathrm{B}}}{2}\left\|\mathbf{g}_{0,0}^{\mathrm{B}}-\mathbf{D}_{0,0} \mathbf{B} \mathbf{f}^{\mathrm{B}}\right\|^{2}\right] & \text { if } c=\mathrm{B} .
\end{array}\right.
\end{aligned}
$$

Note that from the above definitions of the probability density functions, the distribution in (8) depends on a set of unknown parameters and has to be properly written as

$$
\mathbf{p}_{c}\left(\mathbf{f}^{c}, \mathbf{f}^{c^{\prime}}, \mathbf{f}^{c^{\prime \prime}}, \mathbf{g}^{\text {obs } c} \mid \Theta^{c}\right)
$$

where

$$
\Theta^{c}=\left(\alpha^{c}, \gamma^{c c^{\prime}}, \gamma^{c c^{\prime \prime}}, \beta^{c}\right)
$$

Having defined the involved distributions and the unknown parameters, the Bayesian analysis is performed to estimate the parameter vector $\Theta^{c}$ and the unknown highresolution band $\mathbf{f}^{c}$. It is important to remember that we are assuming that $\mathbf{f}^{c^{\prime}}$ and $\mathbf{f}^{c^{\prime \prime}}$ are known.

The process to estimate $\Theta^{c}$ and $\mathbf{f}^{c}$ is described by the following algorithm which corresponds to the so-called evidence analysis within the Bayesian paradigm [27].

Given $\mathbf{f}^{c^{\prime}}$ and $\mathbf{f}^{c^{\prime \prime}}$

(1) Find

$$
\begin{aligned}
\hat{\Theta}^{c}\left(\mathbf{f}^{c^{\prime}}, \mathbf{f}^{c^{\prime \prime}}\right) & =\arg \max _{\Theta^{c}} p_{c}\left(\mathbf{f}^{c^{\prime}}, \mathbf{f}^{c^{\prime \prime}}, \mathbf{g}^{\text {obs } c} \mid \Theta^{c}\right) \\
& =\arg \max _{\Theta^{c}} \int_{\mathbf{f}^{c}} p_{c}\left(\mathbf{f}^{c}, \mathbf{f}^{c^{\prime}}, \mathbf{f}^{c^{\prime \prime}}, \mathbf{g}^{\text {obs } c} \mid \Theta^{c}\right) d \mathbf{f}^{c}
\end{aligned}
$$

(2) Find an estimate of channel $c$ using

$$
\begin{aligned}
& \hat{\mathbf{f}}^{c}\left(\widehat{\Theta}^{c}\left(\mathbf{f}^{c^{\prime}}, \mathbf{f}^{c^{\prime \prime}}\right)\right) \\
& \quad=\arg \max _{\mathbf{f}^{c}} p_{c}\left(\mathbf{f}^{c} \mid \mathbf{f}^{c^{\prime}}, \mathbf{f}^{c^{\prime \prime}}, \mathbf{g}^{\text {obs } c}, \widehat{\Theta}^{c}\left(\mathbf{f}^{c^{\prime}}, \mathbf{f}^{c^{\prime \prime}}\right)\right)
\end{aligned}
$$

Algorithm 1: Estimation of $\Theta^{c}$ and $\mathbf{f}^{c}$ assuming that $\mathbf{f}^{c^{\prime}}$ and $\mathbf{f}^{c^{\prime \prime}}$ are known.

In order to find the hyperparameter vector $\hat{\Theta}^{c}$ and the reconstruction of channel $c$, we use the iterative method described in $[22,23]$.

We now proceed to estimate the whole color image from the incomplete set of observations provided by the singleCCD camera.

Let us assume that we have initial estimates of the three channels $\mathbf{f}^{\mathrm{R}}(0), \mathbf{f}^{\mathrm{G}}(0)$, and $\mathbf{f}^{\mathrm{B}}(0)$; then we can improve the quality of the reconstruction by using the following procedure.
(1) Given $\mathbf{f}^{\mathrm{R}}(0), \mathbf{f}^{\mathrm{G}}(0)$, and $\mathbf{f}^{\mathrm{B}}(0)$, initial estimates of the bands of the color image and $\Theta^{R}(0), \Theta^{G}(0)$, and $\Theta^{B}(0)$ of the model parameters

(2) Set $k=0$

(3) Calculate

$$
\mathbf{f}^{\mathrm{R}}(k+1)=\hat{\mathbf{f}}^{\mathrm{R}}\left(\hat{\Theta}^{\mathrm{R}}\left(\mathbf{f}^{\mathrm{G}}(k), \mathbf{f}^{\mathrm{B}}(k)\right)\right)
$$

by running Algorithm 1 on channel $\mathrm{R}$ with $\mathbf{f}^{\mathrm{G}}=\mathbf{f}^{\mathrm{G}}(k)$ and $\mathbf{f}^{\mathrm{B}}=\mathbf{f}^{\mathrm{B}}(k)$

(4) Calculate

$$
\mathbf{f}^{\mathrm{G}}(k+1)=\hat{\mathbf{f}}^{\mathrm{G}}\left(\hat{\Theta}^{\mathrm{G}}\left(\mathbf{f}^{\mathrm{R}}(k+1), \mathbf{f}^{\mathrm{B}}(k)\right)\right)
$$

by running Algorithm 1 on channel $\mathrm{G}$ with $\mathbf{f}^{\mathrm{R}}=\mathbf{f}^{\mathrm{R}}(k+1)$ and $\mathbf{f}^{\mathrm{B}}=\mathbf{f}^{\mathrm{B}}(k)$

(5) Calculate

$$
\mathbf{f}^{\mathrm{B}}(k+1)=\widehat{\mathbf{f}}^{\mathrm{B}}\left(\widehat{\Theta}^{\mathrm{B}}\left(\mathbf{f}^{\mathrm{R}}(k+1), \mathbf{f}^{\mathrm{G}}(k+1)\right)\right)
$$

by running Algorithm 1 on channel B with $\mathbf{f}^{\mathrm{R}}=\mathbf{f}^{\mathrm{R}}(k+1)$ and $\mathbf{f}^{\mathrm{G}}=\mathbf{f}^{\mathrm{G}}(k+1)$

(6) Set $k=k+1$ and go to step 3 until a convergence criterion is met.

Algorithm 2: Reconstruction of the color image.

\section{ON THE CONSISTENCY OF THE GLOBAL DISTRIBUTION ON THE COLOR IMAGE}

In this section, we examine the use of one global prior distribution on the whole color image instead of using one distribution for each channel.

We could replace the distribution $\mathbf{p}_{c}\left(\mathbf{f}^{c}, \mathbf{f}^{c^{\prime}}, \mathbf{f}^{c^{\prime \prime}}, \mathbf{g}^{\text {obs } c}\right)$ in (8), tailored for channel $c$, by the global distribution

$$
\mathrm{p}\left(\mathbf{f}^{\mathrm{R}}, \mathbf{f}^{\mathrm{G}}, \mathbf{f}^{\mathrm{B}}, \mathbf{g}^{\mathrm{obs}}\right)=\mathrm{p}\left(\mathbf{f}^{\mathrm{R}}, \mathbf{f}^{\mathrm{G}}, \mathbf{f}^{\mathrm{B}}\right) \prod_{c \in\{\mathrm{R}, \mathrm{G}, \mathrm{B}\}} \mathrm{p}_{c}\left(\mathbf{g}^{\mathrm{obs} c} \mid \mathbf{f}^{c}\right),
$$

with

$$
\begin{aligned}
& \mathrm{p}\left(\mathbf{f}^{\mathrm{R}}, \mathbf{f}^{\mathrm{G}}, \mathbf{f}^{\mathrm{B}}\right) \\
& \propto \exp \left\{-\frac{1}{2} \sum_{c \in\{\mathrm{R}, \mathrm{G}, \mathrm{B}\}} \alpha^{c}\left\|C f^{c}\right\|^{2}\right. \\
&\left.-\frac{1}{2} \sum_{c c^{\prime} \in\{\mathrm{RG}, \mathrm{GB}, \mathrm{RB}\}} \sum_{u v \in \mathscr{H} B} \gamma_{u v}^{c c^{\prime}}\left\|W_{u v}\left(\mathbf{f}^{c^{\prime}}-\mathbf{f}^{c}\right)\right\|^{2}\right\},
\end{aligned}
$$

where $W_{u v}$ has been defined in (10), $\alpha^{c}$ measures the smoothness within channel $c$, and $\gamma_{u v}^{c c^{\prime}}$ measures the similarity of the $u v$ band in channels $c$ and $c^{\prime}$ (see (9) and (11)), respectively.

Note that the difference between the models for each channel $c$ in (8) and the one in (21) is that we are not allowing in this new model the case $\gamma_{u v}^{c c^{\prime}} \neq \gamma_{u v}^{c^{\prime} c}$.

We have also used this approach in the experiments. This consistent model can easily be implemented by using Algorithm 2 and forcing $\gamma_{u v}^{c c^{\prime}}=\gamma_{u v}^{c^{\prime} c}$. The results obtained were poorer in terms of improvement in the signal-to-noise 


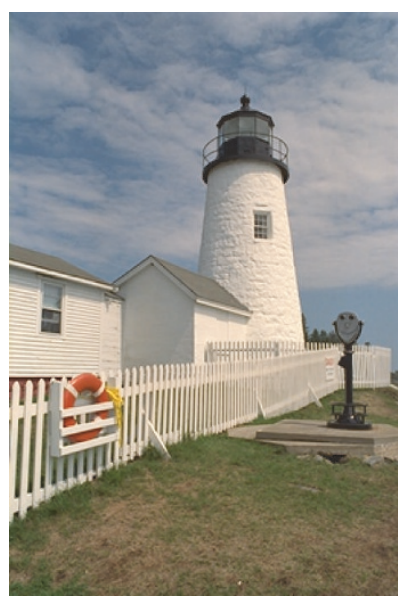

(a)

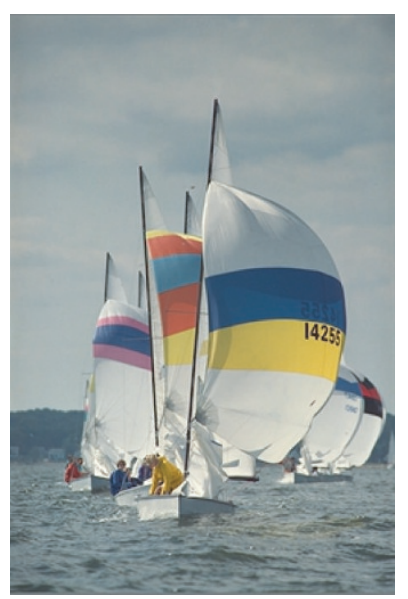

(b)

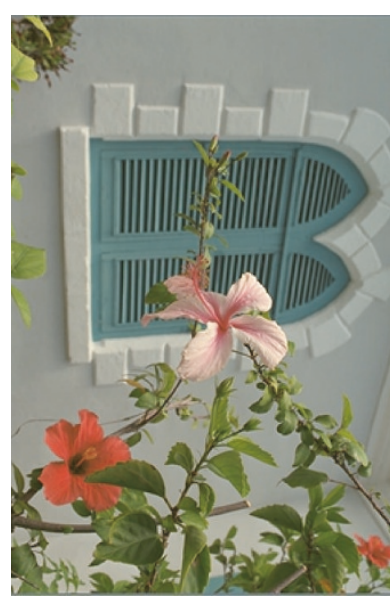

(c)

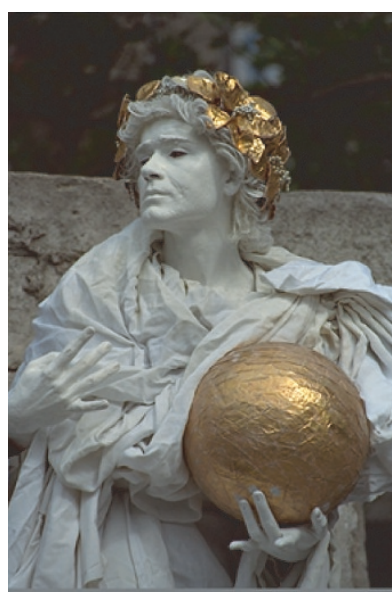

(d)

FIGURE 4: First image set used in the experiments.

ratio. We conjecture that this is due to the fact that the number of observations in each channel is not the same, and therefore each channel has to be responsible for the estimation of the associated hyperparameters.

\section{EXPERIMENTAL RESULTS}

Experiments were carried out with RGB color images in order to evaluate the performance of the proposed method and compare it with other existing ones. Although visual inspection of the restored images is a very important quality measure, in order to get quantitative image quality comparisons, the signal-to-noise ratio improvement $\left(\Delta_{\mathrm{SNR}}\right)$ for each channel is used, given in $\mathrm{dB}$ by

$$
\Delta_{\mathrm{SNR}}^{c}=10 \times \log _{10}\left[\frac{\left\|\mathbf{f}^{c}-\mathbf{g}^{\mathrm{pad} c}\right\|^{2}}{\left\|\mathbf{f}^{c}-\hat{\mathbf{f}}^{c}\right\|^{2}}\right],
$$

for $c \in\{\mathrm{R}, \mathrm{G}, \mathrm{B}\}$, where $\mathbf{f}^{c}$ and $\hat{\mathbf{f}}^{c}$ are the original and estimated high-resolution images, and $\mathbf{g}^{\text {pad } c}$ is the result of padding missing values at the incomplete observed image $\mathbf{g}^{\text {obs } c}$ (3) with zeroes. The mean metric distance $\Delta E_{a b}^{*}$ [28] in the perceptually uniform CIE- $L^{*} a^{*} b^{*}$ color space, between restored and original images, was also used as a figure of merit. In transforming from RGB to CIE- $L^{*} a^{*} b^{*}$ color space, we have used the CIE standard illuminant D65 as reference white and assumed Rec. 709 RGB primaries (see [29]).

Results obtained for two image sets are reported. The first image set is formed by four images of size $256 \times 384$ taken from [6] and shown in Figure 4. Four images of size $640 \times 480$ taken with a 3 CCD color camera (shown in Figure 5) are also used in the experiments.

In order to test the deconvolution method proposed in Algorithm 2, the original images were blurred and then sampled applying a Bayer pattern to get the observed images that were to be reconstructed. Figure 6 illustrates the procedure used to simulate the observation process with a Bayer camera.
It is interesting to observe how blurring and the application of a Bayer pattern interact (see also [21]). Figure 7(a) shows the reconstruction of one CCD observed out-of-focus color image while Figure 7(b) shows the reconstruction of one CCD observed color image (no blur present), using in both cases zero-order hold interpolation. As it can be observed, Figure 7(b) image suffers from the zipper effect in the whole image and exhibits a moire pattern on the wall on the left part of the image. Figure 7(a) shows how blurring may cancel these effects even in the absence of a demosaicing step, at the cost of information loss.

There is not much work reported on the deconvolution of color images acquired with a single sensor. In order to compare our method with others, we have applied a deconvolution step to the output of well-known demosaicing methods. For this deconvolution step, a simultaneous autoregressive (SAR) prior model was used on each channel independently. The underlying idea is that for these methods, the demosaicing step reconstructs, from the incomplete observed $\mathbf{g}^{\text {obs }}$ (3), the blurred image $\mathbf{g}$ that would have been observed with a 3 CCD camera. The degradation model for $\mathbf{f}$ is given by (5). We then performed a Bayesian restoration for every $c$ channel with the probability density

$$
\mathrm{p}_{c}\left(\mathbf{f}^{c}, \mathbf{g}^{c} \mid \alpha^{c}, \beta^{c}\right)=\mathrm{p}_{c}\left(\mathbf{f}^{c} \mid \alpha^{c}\right) \mathrm{p}_{c}\left(\mathbf{g}^{c} \mid \mathbf{f}^{c}, \beta^{c}\right)
$$

with $\mathrm{p}_{c}\left(\mathbf{f}^{c} \mid \alpha^{c}\right)$ given by (9) and (see [27] for details)

$$
\mathrm{p}_{c}\left(\mathbf{g}^{c} \mid \mathbf{f}^{c}, \beta^{c}\right) \propto\left(\beta^{c}\right)^{\left(N_{1} \times N_{2}\right) / 2} \exp \left[-\frac{\beta^{c}}{2}\left\|\mathbf{g}^{c}-\mathbf{B f}^{c}\right\|^{2}\right] \text {. }
$$

Let us now examine the experiments. For the first one, we used an out-of-focus blur with radius $R=2$. The blurring function is given by

$$
h(r) \propto \begin{cases}1 & \text { if } 0 \leq r \leq R, \\ 0 & \text { if } r>R\end{cases}
$$

with normalization needed for conserving the image flux. 


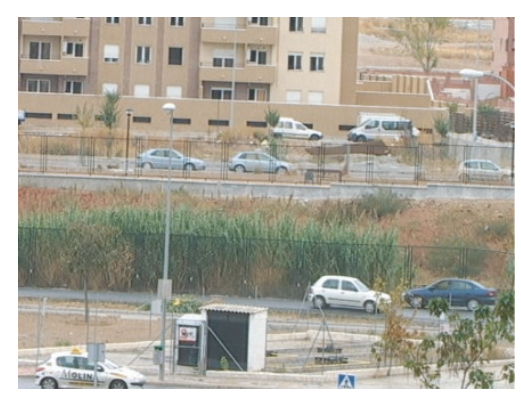

(a)

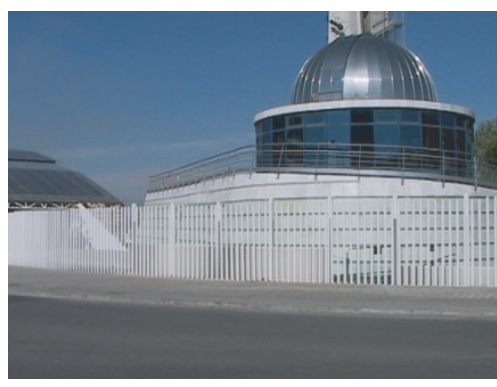

(c)

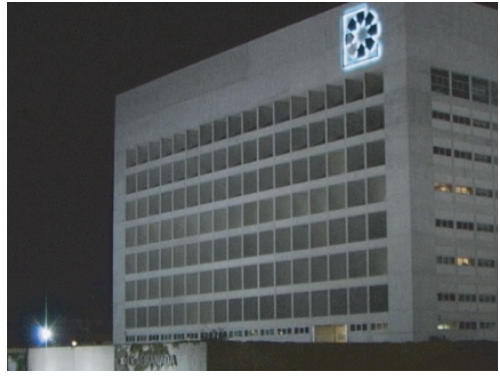

(b)

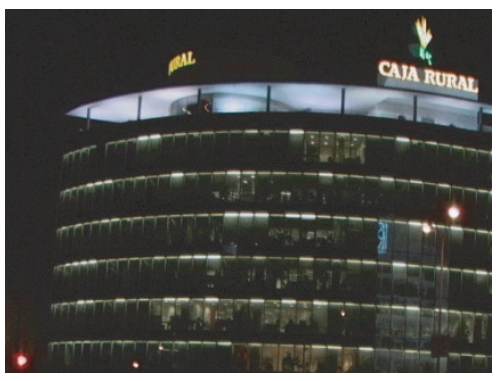

(d)

FIGURE 5: Second image set used in the experiments.

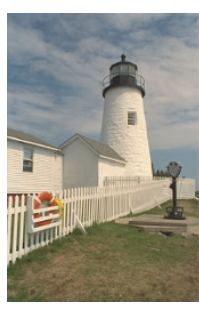

Original image

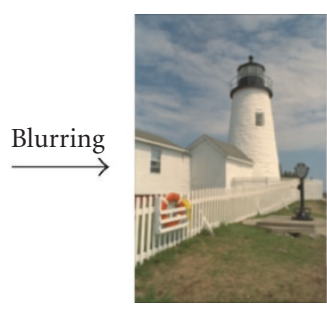

FIGURE 6: Observation process of a blurred image using a Bayer camera.

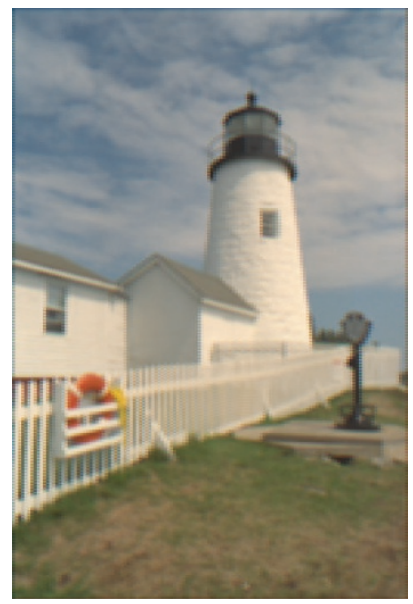

(a)

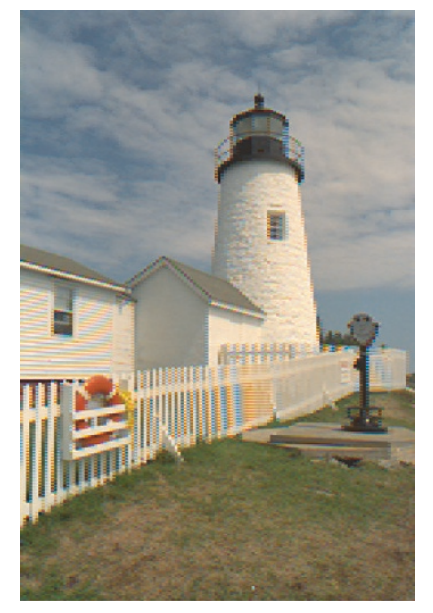

(b)

FIGURE 7: (a) Zero-order hold reconstruction with blur present, and (b) without blur. 


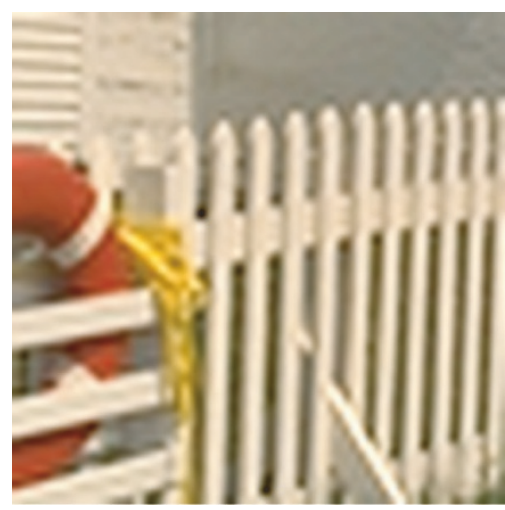

(a)

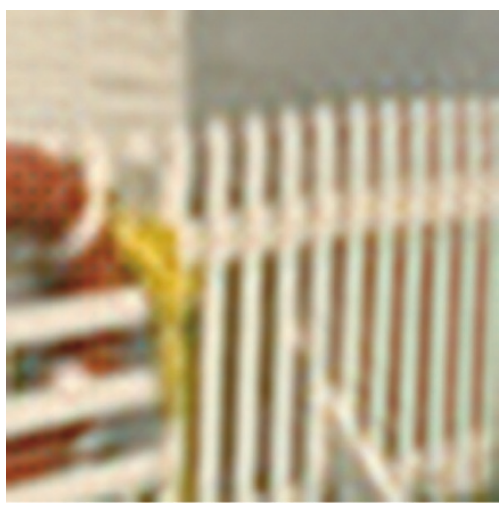

(d)

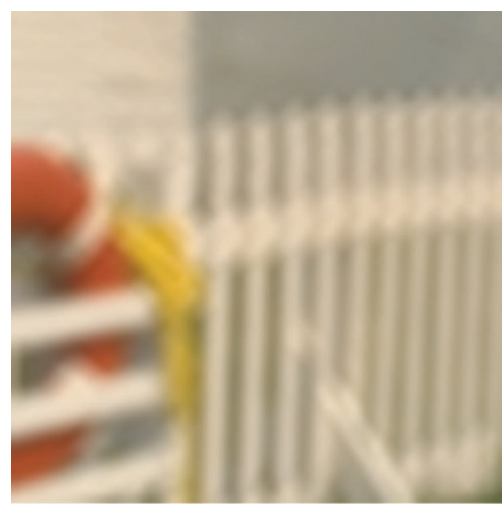

(b)

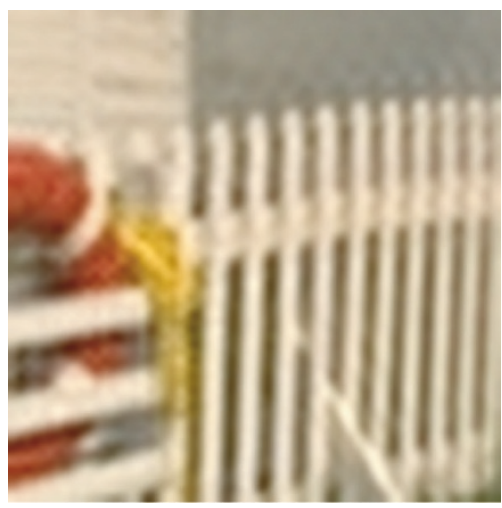

(e)

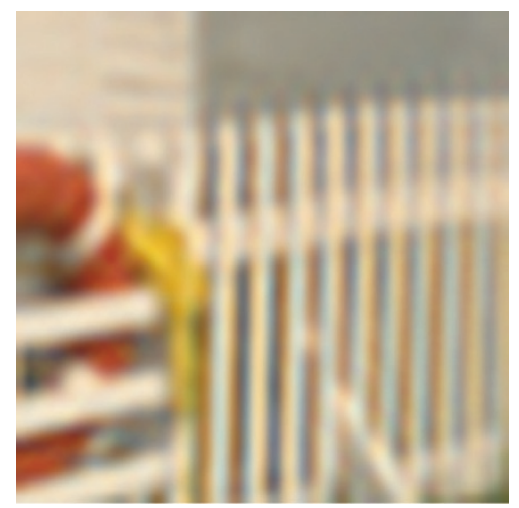

(c)

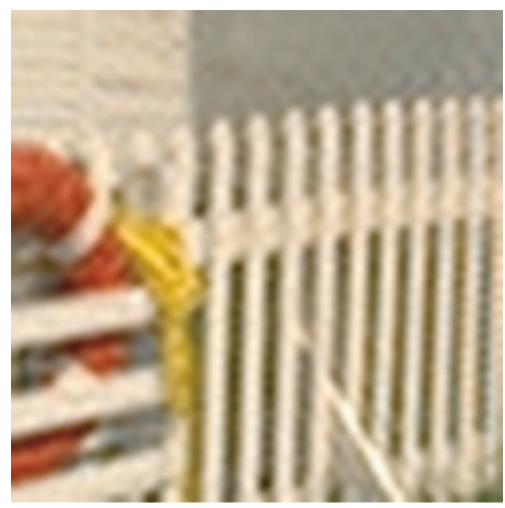

(f)

FIGURE 8: (a) Details of the original image of Figure 4(a), (b) blurred image, (c) deconvolution after applying bilinear reconstruction, (d) deconvolution after applying the method of Laroche and Prescott [5], (e) deconvolution after applying the method of Gunturk et al. [7], and (f) our method.

TABLE 1: Out-of-focus deblurring $\Delta_{\mathrm{SNR}}(\mathrm{dB})$.

\begin{tabular}{|c|c|c|c|c|}
\hline $\begin{array}{l}\text { Original } \\
\text { image }\end{array}$ & Bilinear & $\begin{array}{l}\text { Laroche and } \\
\text { Prescott [5] }\end{array}$ & $\begin{array}{l}\text { Gunturk } \\
\text { et al. [7] }\end{array}$ & $\begin{array}{c}\text { Our } \\
\text { method }\end{array}$ \\
\hline Figure $4(a)^{\mathrm{R}}$ & 18.1 & 18.0 & 19.6 & 21.5 \\
\hline Figure $4(a)^{\mathrm{G}}$ & 16.7 & 17.0 & 17.4 & 19.4 \\
\hline Figure $4(a)^{B}$ & 16.4 & 17.4 & 18.1 & 19.9 \\
\hline Figure $4(b)^{\mathrm{R}}$ & 20.9 & 20.8 & 22.8 & 24.7 \\
\hline Figure $4(b)^{\mathrm{G}}$ & 20.6 & 20.8 & 21.1 & 23.5 \\
\hline Figure $4(b)^{B}$ & 20.8 & 22.1 & 22.2 & 24.5 \\
\hline Figure $4(\mathrm{c})^{\mathrm{R}}$ & 19.6 & 18.8 & 21.8 & 24.6 \\
\hline Figure $4(\mathrm{c})^{\mathrm{G}}$ & 18.8 & 19.1 & 19.6 & 22.3 \\
\hline Figure $4(\mathrm{c})^{\mathrm{B}}$ & 17.2 & 18.4 & 19.7 & 21.8 \\
\hline Figure $4(d)^{R}$ & 18.4 & 18.0 & 18.2 & 22.3 \\
\hline Figure $4(\mathrm{~d})^{\mathrm{G}}$ & 17.0 & 17.1 & 17.6 & 20.3 \\
\hline Figure $4(d)^{B}$ & 16.9 & 18.2 & 18.3 & 20.9 \\
\hline Figure $5(a)^{\mathrm{R}}$ & 21.2 & 21.8 & 24.9 & 25.4 \\
\hline Figure $5(a)^{\mathrm{G}}$ & 20.6 & 22.4 & 23.1 & 23.3 \\
\hline Figure $5(a)^{B}$ & 19.8 & 23.1 & 23.4 & 23.3 \\
\hline Figure $5(b)^{R}$ & 21.2 & 23.3 & 25.1 & 25.5 \\
\hline Figure $5(b)^{G}$ & 21.5 & 23.2 & 23.9 & 24.0 \\
\hline Figure $5(b)^{B}$ & 21.9 & 25.2 & 25.8 & 25.1 \\
\hline Figure $5(\mathrm{c})^{\mathrm{R}}$ & 22.3 & 21.8 & 23.4 & 26.2 \\
\hline Figure $5(\mathrm{c})^{\mathrm{G}}$ & 22.8 & 21.8 & 21.9 & 25.4 \\
\hline Figure $5(\mathrm{c})^{\mathrm{B}}$ & 22.2 & 23.3 & 23.6 & 27.2 \\
\hline Figure $5(\mathrm{~d})^{\mathrm{R}}$ & 18.7 & 19.8 & 22.2 & 24.5 \\
\hline Figure $5(d)^{G}$ & 18.9 & 20.2 & 21.0 & 23.1 \\
\hline Figure $5(d)^{B}$ & 18.5 & 21.4 & 22.2 & 24.4 \\
\hline
\end{tabular}

TABLE 2: Out-of-focus deblurring $\Delta E_{a b}^{*}$.

\begin{tabular}{ccccc}
\hline $\begin{array}{c}\text { Original } \\
\text { image }\end{array}$ & Bilinear & $\begin{array}{c}\text { Laroche and } \\
\text { Prescott [5] }\end{array}$ & $\begin{array}{c}\text { Gunturk } \\
\text { et al. [7] }\end{array}$ & $\begin{array}{c}\text { Our } \\
\text { method }\end{array}$ \\
\hline Figure 4(a) & 3.0 & 3.5 & 2.8 & 2.2 \\
Figure 4(b) & 1.9 & 2.4 & 2.0 & 1.4 \\
Figure 4(c) & 3.3 & 3.8 & 2.9 & 2.2 \\
Figure 4(d) & 3.2 & 3.7 & 3.2 & 2.6 \\
Figure 5(a) & 2.4 & 2.3 & 1.6 & 1.4 \\
Figure 5(b) & 4.5 & 5.3 & 5.2 & 3.6 \\
Figure 5(c) & 1.6 & 2.9 & 2.9 & 1.1 \\
Figure 5(d) & 8.1 & 13.4 & 14.7 & 7.4 \\
\hline
\end{tabular}

Figure 8 shows the image of Figure 4(a) and its blurred observation, just before the application of the Bayer pattern. Figure 8 shows also the reconstruction obtained by bilinear interpolation followed by deconvolution, and deconvolutions of the results of demosaicing the blurred image with the methods proposed by Laroche and Prescott [5] and Gunturk et al. [7]. Figure 8(f) shows the result obtained with the application of Algorithm 2. Figure 8 shows how demosaicing may introduce the undesirable effects that blurring had cancelled. This fact is more noticeable for bilinear interpolation but remains in the Laroche and Prescott method [5]. The method of [7] is very efficient in demosaicing, but our method gives better results in demosaicing while recovering 


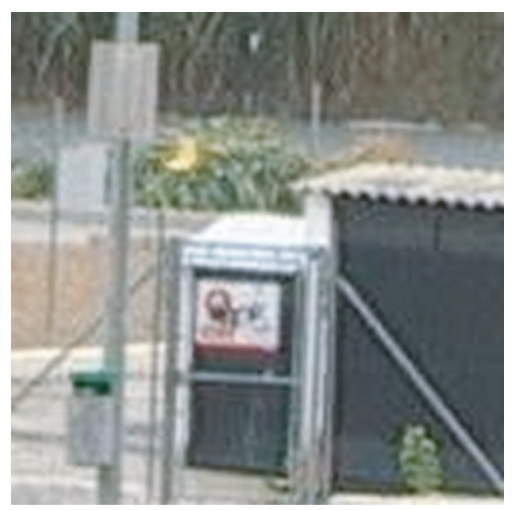

(a)

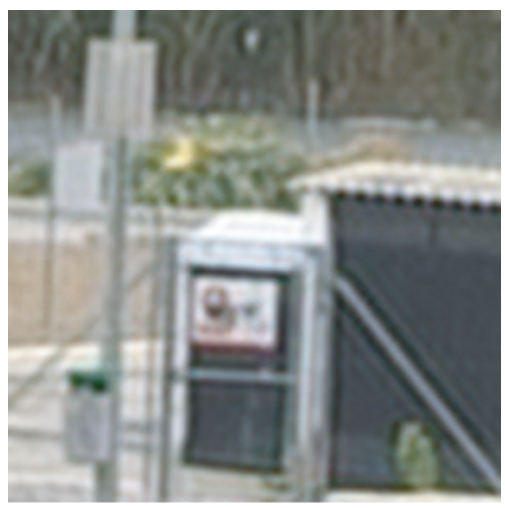

(d)

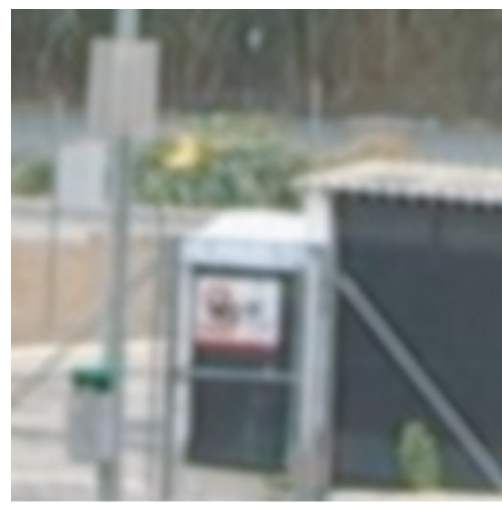

(b)

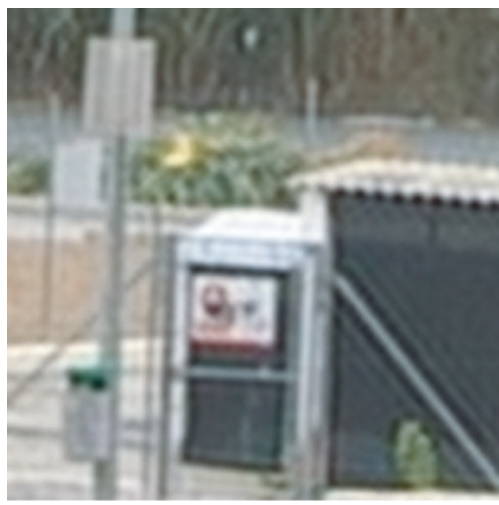

(e)

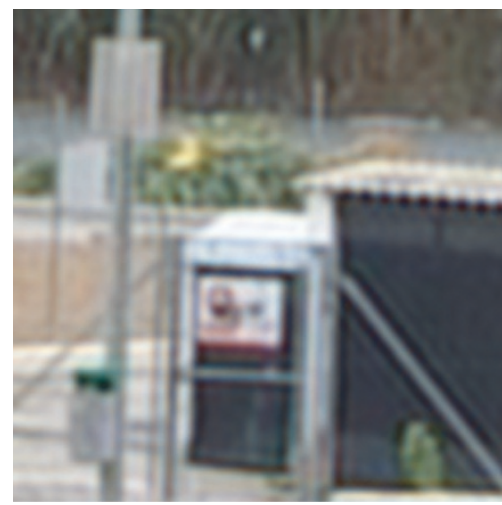

(c)

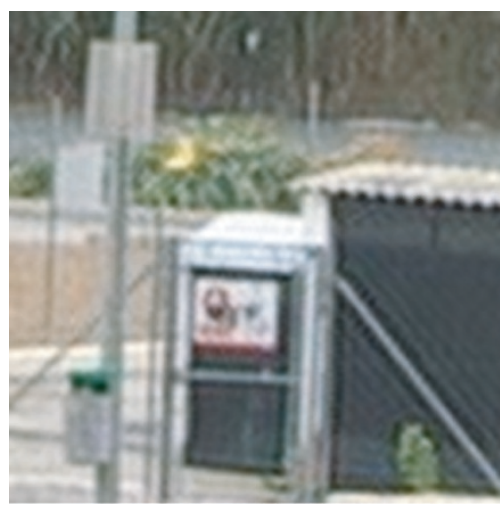

(f)

FIGURE 9: (a) Details of the original image of Figure 5(a), (b) out-of-focus image, (c) deconvolution after applying bilinear reconstruction, (d) deconvolution after applying the method of Laroche and Prescott [5], (e) deconvolution after applying the method of Gunturk et al. [7], and (f) our method.

TABLE 3: Motion deblurring $\Delta_{\mathrm{SNR}}(\mathrm{dB})$.

\begin{tabular}{|c|c|c|c|c|}
\hline $\begin{array}{l}\text { Original } \\
\text { image }\end{array}$ & Bilinear & $\begin{array}{l}\text { Laroche and } \\
\text { Prescott [5] }\end{array}$ & $\begin{array}{l}\text { Gunturk } \\
\text { et al. [7] }\end{array}$ & $\begin{array}{c}\text { Our } \\
\text { method }\end{array}$ \\
\hline Figure $4(a)^{\mathrm{R}}$ & 18.1 & 17.1 & 17.9 & 22.8 \\
\hline Figure $4(a)^{\mathrm{G}}$ & 18.4 & 15.8 & 15.6 & 21.1 \\
\hline Figure $4(a)^{B}$ & 16.3 & 16.4 & 16.7 & 21.2 \\
\hline Figure $4(b)^{\mathrm{R}}$ & 21.0 & 19.1 & 19.9 & 26.4 \\
\hline Figure $4(b)^{G}$ & 22.6 & 19.0 & 18.6 & 25.6 \\
\hline Figure $4(b)^{\mathrm{B}}$ & 21.0 & 19.8 & 19.8 & 26.3 \\
\hline Figure $4(\mathrm{c})^{\mathrm{R}}$ & 20.1 & 17.0 & 19.4 & 27.0 \\
\hline Figure $4(\mathrm{c})^{\mathrm{G}}$ & 21.1 & 17.4 & 17.3 & 25.3 \\
\hline Figure $4(\mathrm{c})^{\mathrm{B}}$ & 17.5 & 17.3 & 18.0 & 23.8 \\
\hline Figure $4(\mathrm{~d})^{\mathrm{R}}$ & 19.0 & 16.9 & 17.4 & 24.9 \\
\hline Figure $4(\mathrm{~d})^{\mathrm{G}}$ & 19.3 & 16.1 & 15.7 & 23.6 \\
\hline Figure $4(d)^{B}$ & 17.0 & 16.9 & 16.8 & 24.0 \\
\hline Figure $5(a)^{R}$ & 21.0 & 19.7 & 22.6 & 25.6 \\
\hline Figure $5(a)^{\mathrm{G}}$ & 21.7 & 20.6 & 20.7 & 23.8 \\
\hline Figure $5(a)^{B}$ & 19.6 & 21.5 & 21.5 & 24.0 \\
\hline Figure $5(b)^{R}$ & 20.7 & 21.4 & 22.6 & 24.6 \\
\hline Figure $5(b)^{G}$ & 22.0 & 21.0 & 21.1 & 23.5 \\
\hline Figure $5(b)^{B}$ & 21.4 & 22.6 & 22.8 & 24.6 \\
\hline Figure $5(\mathrm{c})^{\mathrm{R}}$ & 21.6 & 20.3 & 23.4 & 23.7 \\
\hline Figure $5(\mathrm{c})^{\mathrm{G}}$ & 22.4 & 22.0 & 21.8 & 22.7 \\
\hline Figure $5(c)^{B}$ & 21.4 & 23.2 & 23.3 & 23.8 \\
\hline Figure $5(d)^{R}$ & 18.2 & 17.5 & 20.3 & 23.3 \\
\hline Figure $5(d)^{G}$ & 19.9 & 18.8 & 18.7 & 21.9 \\
\hline Figure $5(d)^{B}$ & 18.0 & 20.2 & 20.2 & 22.9 \\
\hline
\end{tabular}

TABLE 4: Motion deblurring $\Delta E_{a b}^{*}$.

\begin{tabular}{ccccc}
\hline $\begin{array}{c}\text { Original } \\
\text { image }\end{array}$ & Bilinear & $\begin{array}{c}\text { Laroche and } \\
\text { Prescott [5] }\end{array}$ & $\begin{array}{c}\text { Gunturk } \\
\text { et al. [7] }\end{array}$ & $\begin{array}{c}\text { Our } \\
\text { method }\end{array}$ \\
\hline Figure 4(a) & 3.7 & 4.2 & 3.1 & 1.9 \\
Figure 4(b) & 2.3 & 3.0 & 2.4 & 1.2 \\
Figure 4(c) & 3.8 & 4.0 & 3.2 & 1.9 \\
Figure 4(d) & 3.7 & 4.9 & 4.5 & 2.1 \\
Figure 5(a) & 3.0 & 3.4 & 1.8 & 1.3 \\
Figure 5(b) & 4.9 & 6.1 & 6.0 & 3.3 \\
Figure 5(c) & 1.8 & 2.4 & 1.6 & 1.4 \\
Figure 5(d) & 8.8 & 13.2 & 13.8 & 6.9 \\
\hline
\end{tabular}

the information lost with blurring, probably at the cost of a light aliasing effect.

Table 1 compares, in terms of $\Delta_{\mathrm{SNR}}$, the results obtained by deconvolved bilinear interpolation and by the abovementioned methods to deconvolve single-CCD observed color images. Table 2 compares the results obtained in terms of $\Delta E_{a b}^{*}$ color differences. Figure 9 shows details corresponding to the reconstruction of Figure 5(a), and Figure 10 shows the reconstructions corresponding to Figure 5(c). It can be observed that in all cases, the proposed method produces better reconstructions both in terms of perceptual quality $\Delta E_{a b}^{*}$ and $\Delta_{S N R}^{c}$ values. Figure 11 shows the convergence rate of Algorithm 2 in the reconstruction of an image from the first set (see Figure 4(a)). 


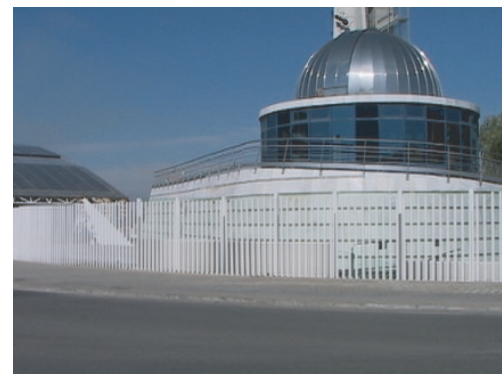

(a)

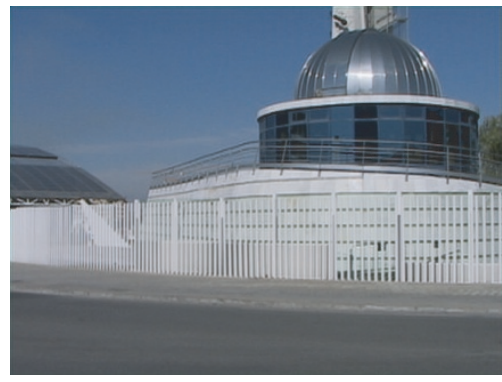

(d)

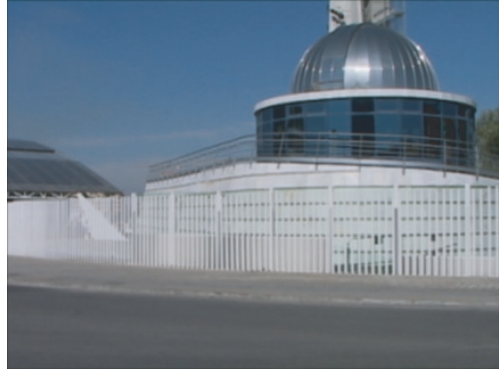

(b)

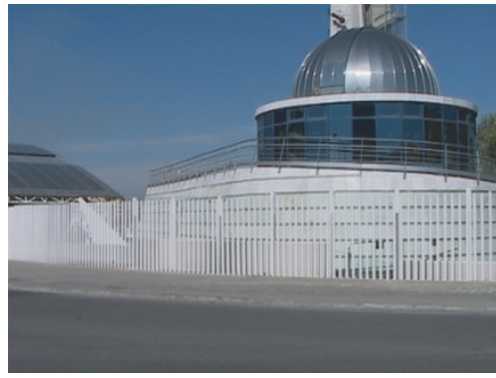

(e)

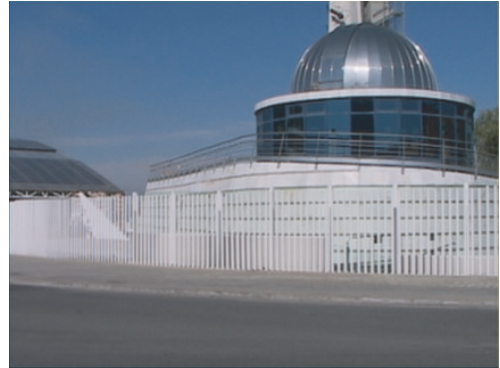

(c)

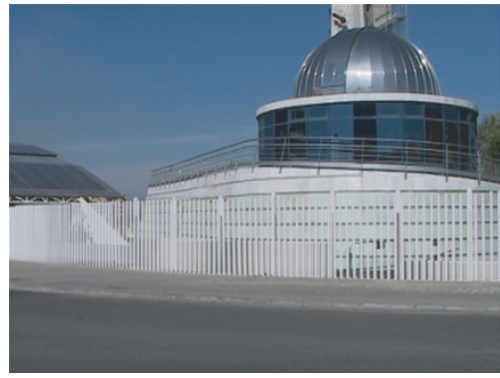

(f)

FIGURE 10: (a) Original image of Figure 5(c), (b) out-of-focus image, (c) deconvolution after applying bilinear reconstruction, (d) deconvolution after applying the method of Laroche and Prescott [5], (e) deconvolution after applying the method of Gunturk et al. [7], and (f) our method.

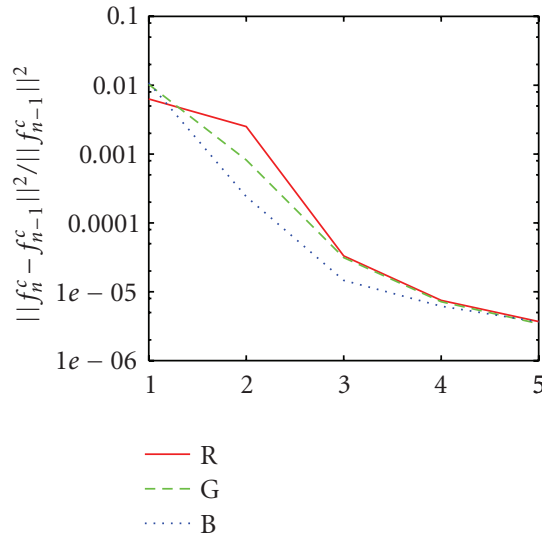

(a)
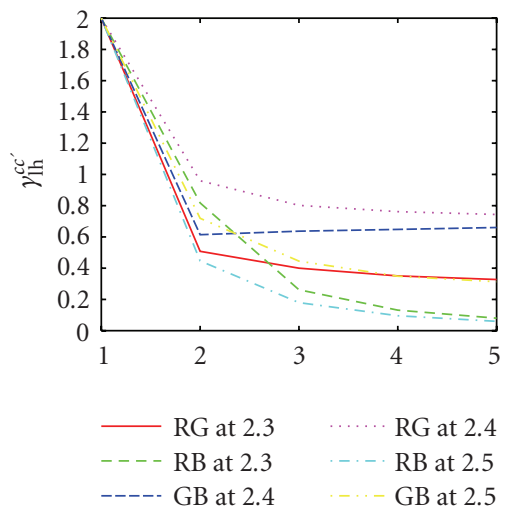

(d)
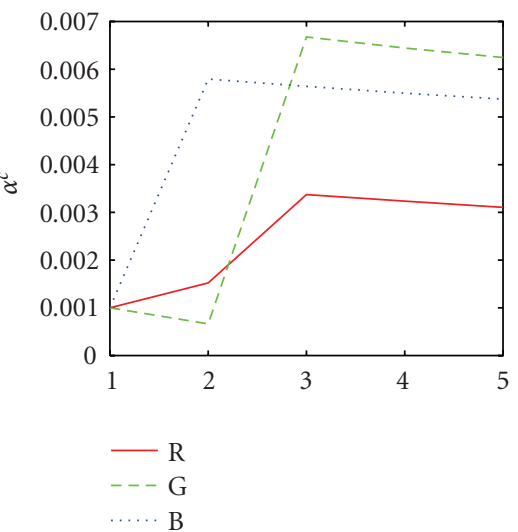

(b)

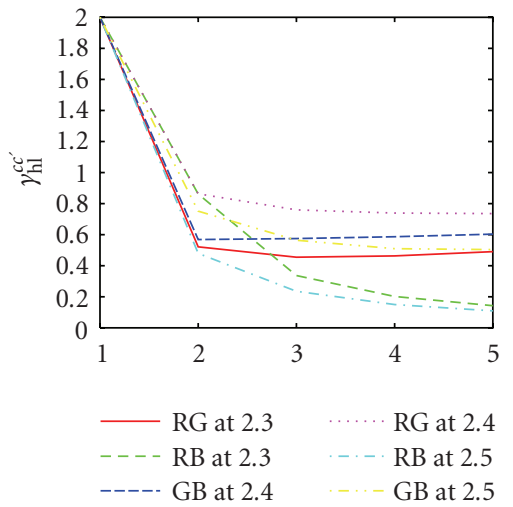

(e)

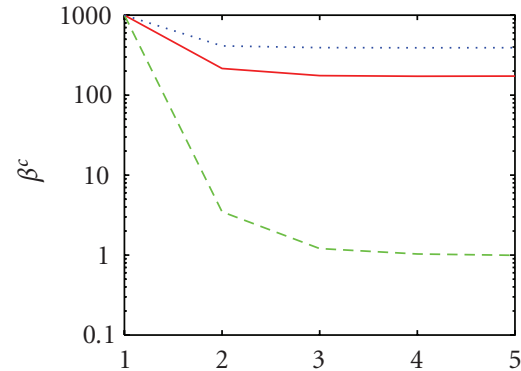

- R

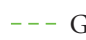

…. B

(c)

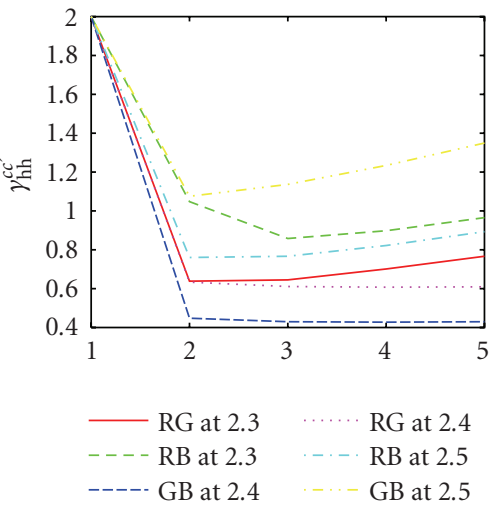

(f)

FIGURE 11: Several plots (a) convergence rate, (b) $\alpha^{c}$, (c) $\beta^{c}$, (d) $\gamma_{l h}^{c c}$, (e) $\gamma_{h l}^{c c l}$, and (f) $\gamma_{h h}^{c c l}$ versus iterations corresponding to the application of Algorithm 2 to the reconstruction of the image of Figure 4(a), for out-of-focus blurring. 


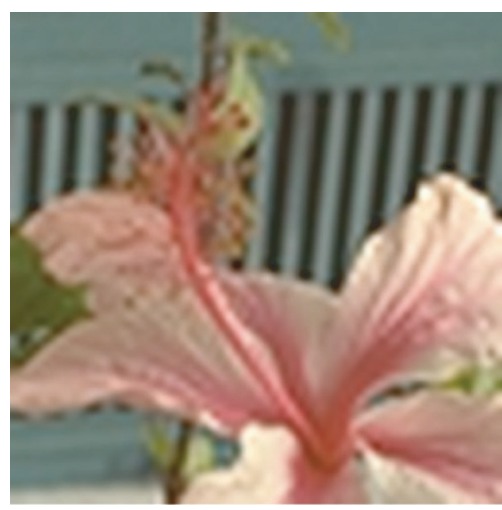

(a)

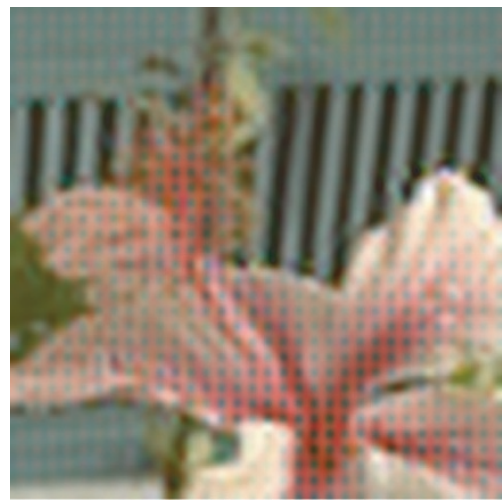

(d)

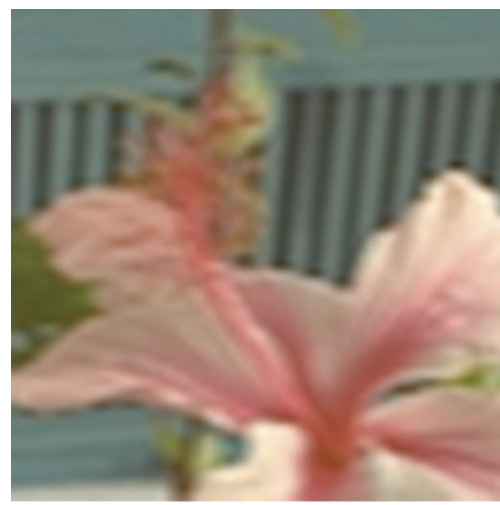

(b)

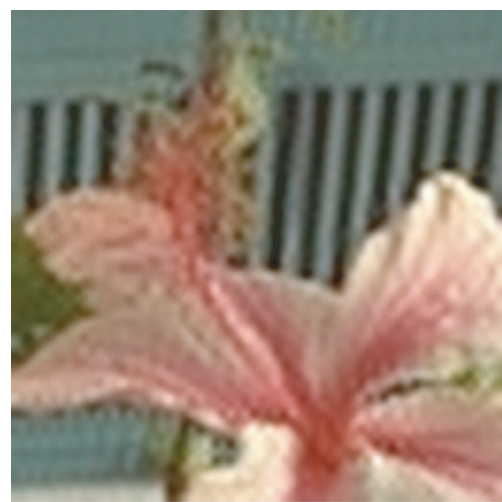

(e)

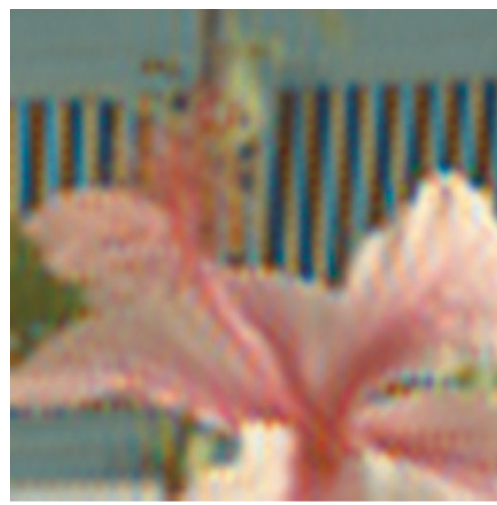

(c)

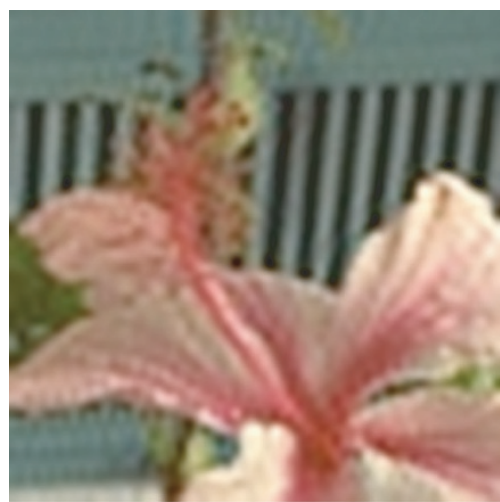

(f)

Figure 12: (a) Details of the original image of Figure 4(c), (b) image blurred with horizontal motion, (c) deconvolution after applying bilinear reconstruction, (d) deconvolution after applying the method of Laroche and Prescott [5], (e) deconvolution after applying the method of Gunturk et al. [7], and (f) our method.

In the second experiment, we investigated the behavior of our method under motion blur. The blurring function used is given by

$$
h(x, y)= \begin{cases}\frac{1}{L} & \text { if }(0 \leq x<L),(y=0), \\ 0 & \text { otherwise, }\end{cases}
$$

$L$ is the displacement by the horizontal motion. A displacement of $L=3$ pixels was used. A Bayer pattern was also applied to the images, as in the first experiment.

Table 3 compares the $\Delta_{S N R}^{c}$ values obtained by the above mentioned methods to deconvolve single-CCD observed color images for the different images under consideration. Table 4 compares the results obtained in terms of $\Delta E_{a b}^{*}$ color differences. Figures 12 and 13 show details of the images of Figures 4(d) and 5(b), respectively, their observations, and their corresponding restorations. Algorithm 2 obtains, in this case again, better reconstructions than deconvolved bilinear interpolation and the methods in [5] and [7], based on visual examination, and in the numeric values in Tables 3 and 4.

In all experiments, the proposed Algorithm 2 was run using as initial image estimates bilinearly interpolated images, and the initial values $\alpha^{c(0)}=0.001, \beta^{c(0)}=1000.0$, and $\gamma_{u v}^{c c^{\prime}(0)}=2.0$ (for all $u v \in \mathscr{H} B$ and $c^{\prime} \neq c$ ) for all $c \in\{R, G, B\}$. The convergence criterion utilized was

$$
\frac{\left\|\mathbf{f}^{c}(k+1)-\mathbf{f}^{c}(k)\right\|^{2}}{\left\|\mathbf{f}^{c}(k)\right\|^{2}} \leq \epsilon
$$

with values for $\epsilon$ between $10^{-5}$ and $10^{-7}$.

It has been very helpful for the elaboration of this experimental section the description in [2] of the method in [5], and the code for the method in [7] accessible in [30].

\section{CONCLUSIONS}

In this paper, the deconvolution problem of color images acquired with a single sensor has been formulated from a super-resolution point of view. A new method for estimating both the reconstructed color images and the model parameters, within the Bayesian framework, was obtained. Based on the presented experimental results, the new method outperforms the application of deconvolution techniques to wellestablished demosaicing methods. 


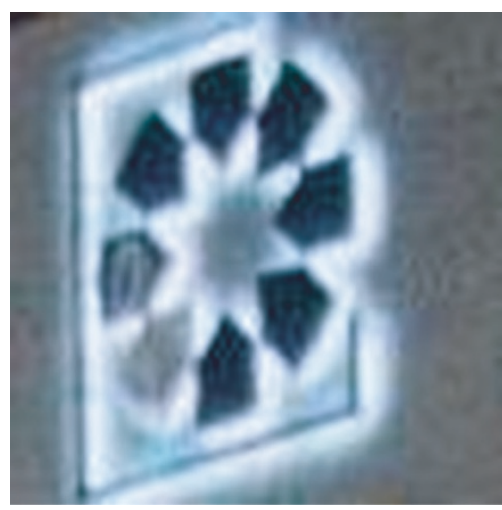

(a)

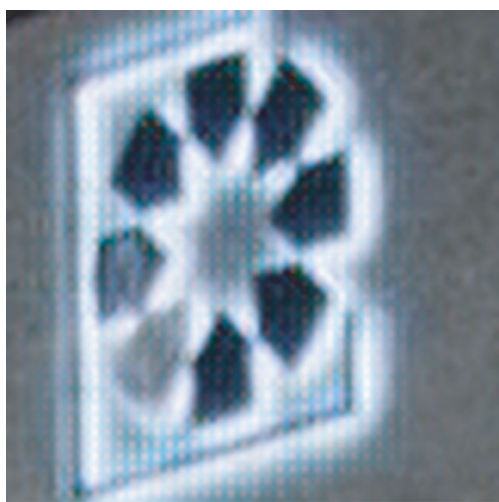

(d)

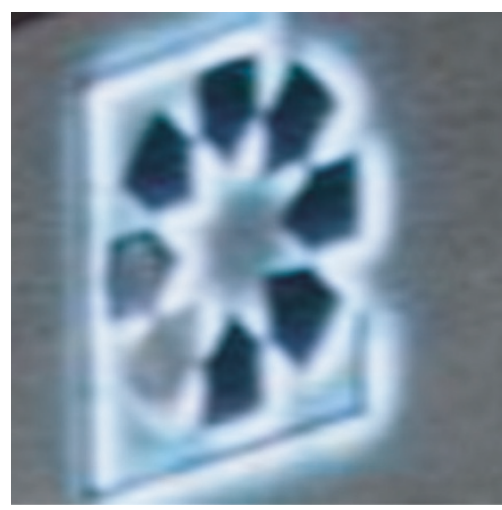

(b)

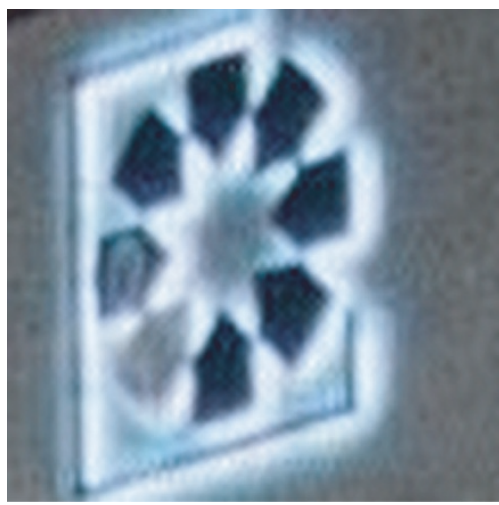

(e)

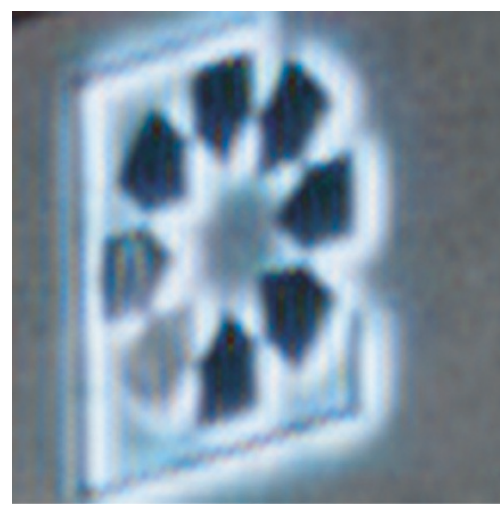

(c)

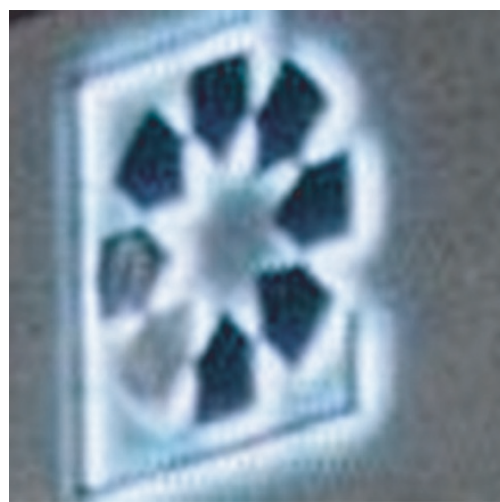

(f)

Figure 13: (a) Details of the original image of Figure 5(b), (b) image blurred with horizontal motion, (c) deconvolution after applying bilinear reconstruction, (d) deconvolution after applying the method of Laroche and Prescott [5], (e) deconvolution after applying the method of Gunturk et al. [7], and (f) our method.

\section{ACKNOWLEDGMENT}

This work has been supported by the "Comisión Nacional de Ciencia y Tecnología” under Contract TIC2003-00880.

\section{REFERENCES}

[1] B. E. Bayer, "Color imaging array," 1976, United States Patent $3,971,065$.

[2] R. Ramanath, "Interpolation methods for the Bayer color array," Ph.D. dissertation, North Carolina State University, Raleigh, NC, USA, 2000.

[3] D. R. Cok, "Signal processing method and apparatus for producing interpolated chrominance values in a sampled color image signal," 1987, United States Patent 4,642,678.

[4] T. W. Freeman, "Median filter for reconstructing missing color samples," 1988, United States Patent 4,724,395.

[5] C. A. Laroche and M. A. Prescott, "Apparatus and method for adaptively interpolating a full color image utilizing chrominance gradients," 1994, United States Patent 5,373,322.

[6] R. kimmel, "Demosaicing: image reconstruction from color CCD samples," IEEE Transactions Image Processing, vol. 8, no. 9, pp. 1221-1228, 1999.

[7] B. K. Gunturk, Y. Altunbasak, and R. M. Mersereau, "Color plane interpolation using alternating projections," IEEE Transactions Image Processing, vol. 11, no. 9, pp. 997-1013, 2002.
[8] P. Scheunders, "An orthogonal wavelet representation of multivalued images," IEEE Transactions Image Processing, vol. 12, no. 6, pp. 718-725, 2003.

[9] L. D. Alvarez, R. Molina, and A. K. Katsaggelos, "High resolution images from a sequence of low resolution observations," in Digital Image Sequence Processing, Compression and Analysis, T. R. Reed, Ed., chapter 9, pp. 233-259, CRC Press, Boca Raton, Fla, USA, 2004.

[10] M. K. Ng, R. H. Chan, T. F. Chan, and A. M. Yip, "Cosine transform preconditioners for high resolution image reconstruction," Linear Algebra and its Applications, vol. 316, no. 13, pp. 89-104, 2000.

[11] N. Nguyen and P. Milanfar, "A wavelet-based interpolationrestoration method for superresolution," Circuits, Systems, and Signal Processing, vol. 19, no. 4, pp. 321-338, 2000.

[12] N. Nguyen, P. Milanfar, and G. Golub, "A computationally efficient superresolution image reconstruction algorithm," IEEE Transactions Image Processing, vol. 10, no. 4, pp. 573-583, 2001.

[13] M. K. Ng and A. M. Yip, "A fast MAP algorithm for highresolution image reconstruction with multisensors," Multidimensional Systems and Signal Processing, vol. 12, no. 2, pp. 143-164, 2001.

[14] M. G. Kang and S. Chaudhuri, "Super-resolution image reconstruction,” IEEE Signal Processing Magzine, vol. 20, no. 3, pp. 19-20, 2003. 
[15] N. K. Bose, R. H. Chan, and M. K. Ng, "Special issue on highresolution image reconstruction. I. Guest editorial," International Journal of Imaging Systems and Technology, vol. 14, no. 2, pp. 35-35, 2004.

[16] E. Choi, J. Choi, and M. G. Kang, "Super-resolution approach to overcome physical limitations of imaging sensors: an overview," International Journal of Imaging Systems and Technology, vol. 14, no. 2, pp. 36-46, 2004.

[17] S. Farsiu, D. Robinson, M. Elad, and P. Milanfar, "Advances and challenges in super-resolution," International Journal of Imaging Systems and Technology, vol. 14, no. 2, pp. 47-57, 2004.

[18] A. Zomet and S. Peleg, "Multi-sensor super-resolution," in Proceedings of 6th IEEE Workshop on Applications of Computer Vision (WACV'02), pp. 27-31, Orlando, Fla, USA, December 2002.

[19] S. Farsiu, M. Elad, and P. Milanfar, "Multiframe demosaicing and super-resolution from undersampled color images," in Computational Imaging II, vol. 5299 of Proceedings of SPIE, pp. 222-233, San Jose, Calif, USA, January 2004.

[20] T. Gotoh and M. Okutomi, "Direct super-resolution and registration using raw CFA images," in Proceedings of IEEE Computer Society Conference on Computer Vision and Pattern Recognition (CVPR '04), vol. 2, pp. 600-607, Washington, DC, USA, June-July 2004.

[21] S. Farsiu, M. Elad, and P. Milanfar, "Multi-frame demosaicing and super-resolution of color images," IEEE Transactions Image Processing, vol. 15, no. 1, pp. 141-159, 2006.

[22] J. Mateos, R. Molina, and A. K. Katsaggelos, "Bayesian high resolution image reconstruction with incomplete multisensor low resolution systems," in Proceedings of IEEE International Conference on Acoustics, Speech, and Signal Processing (ICASSP '03), vol. 3, pp. 705-708, Hong Kong, April 2003.

[23] R. Molina, M. Vega, J. Abad, and A. K. Katsaggelos, "Parameter estimation in Bayesian high-resolution image reconstruction with multisensors," IEEE Transactions Image Processing, vol. 12, no. 12, pp. 1655-1667, 2003.

[24] A. K. Katsaggelos, K. T. Lay, and N. P. Galatsanos, "A general framework for frequency domain multi-channel signal processing," IEEE Transactions Image Processing, vol. 2, no. 3, pp. 417-420, 1993.

[25] R. Molina, J. Mateos, A. K. Katsaggelos, and M. Vega, "Bayesian multichannel image restoration using compound Gauss-Markov random fields," IEEE Transactions Image Processing, vol. 12, no. 12, pp. 1642-1654, 2003.

[26] J. Mateos, M. Vega, R. Molina, and A. K. Katsaggelos, "Bayesian image estimation from an incomplete set of blurred, undersampled low resolution images," in Proceedings of $1 \mathrm{st}$ Iberian Conference on Pattern Recognition and Image Analysis (IbPRIA '03), vol. 2652 of Lecture Notes in Computer Science, pp. 538-546, Puerto de Andratx, Mallorca, Spain, June 2003.

[27] R. Molina, A. K. Katsaggelos, and J. Mateos, "Bayesian and regularization methods for hyperparameter estimation in image restoration," IEEE Transactions Image Processing, vol. 8, no. 2, pp. 231-246, 1999.

[28] Commission Internationale de L'Éclairage, Colorimetry, CIE, Vienna, Austria, 2nd edition, 1986, publication CIE no. 15.2.

[29] International Telecommunication Union, Basic Parameter Values for the HDTV Standard for the Studio and for International Programme Exchange, ITU, Geneva, Switzerland, 1990, ITU-R Recommendation BT.709.

[30] Y. Altunbasak, 2002, available at: http://www.ece.gatech.edu/ research/labs/MCCL/research/topic05.html.
Miguel Vega was born 1956 in Spain. He received his Bachelor Physics degree from Universidad de Granada (1979) and Ph.D. degree from Universidad de Granada (Departmento de Física Nuclear, 1984). He is a staff member (1984-1987) and Director (1989-1992) of the Computing Center Facility of Universidad de Granada. He is a Lecturer 1987 till now in the ETS Ingeriería Informática of Universidad de

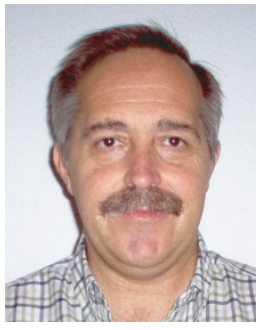
Granada (Departmento de Lenguajes y Sistemas Informáticos). He teaches software engineering. His research focuses on image processing (multichannel and super-resolution image reconstruction). He has collaborated at several projects from the Spanish Research Council.

Rafael Molina was born in 1957. He received the degree in mathematics (statistics) in 1979 and the Ph.D. degree in optimal design in linear models in 1983. He became Professor of computer science and artificial intelligence at the University of Granada, Granada, Spain, in 2000. His areas of research interest are image restoration (applications to astronomy and medicine), parameter estimation in image restoration,

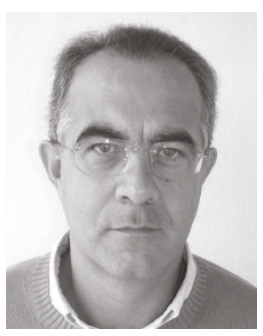
low-to-high image and video, and blind deconvolution. Dr. Molina is a Member of SPIE, Royal Statistical Society, and the Asociación Española de Reconocimiento de Formas y Análisis de Imágenes (AERFAI).

Aggelos K. Katsaggelos received the Diploma degree in electrical and mechanical engineering from the Aristotelian University of Thessaloniki, Greece, in 1979 and the M.S. and Ph.D. degrees both in electrical engineering from the Georgia Institute of Technology, in 1981 and 1985, respectively. He is currently a Professor of electrical engineering and computer science at Northwestern University and also the Di-

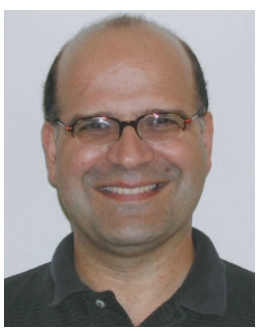
rector of the Motorola Center for Seamless Communications and a Member of the academic affiliate staff, Department of Medicine, at Evanston Hospital. He is the Editor of Digital Image Restoration (New York, Springer, 1991), coauthor of Rate-Distortion Based Video Compression (Kluwer, Norwell, 1997), and coeditor of Recovery Techniques for Image and Video Compression and Transmission (Kluwer, Norwell, 1998), and the coinventor of ten international patents. Dr. Katsaggelos is a Member of the Publication Board of the IEEE Proceedings, and has served as the Editor-in-Chief of the IEEE Signal Processing Magazine (19972002), He is the recipient of the IEEE Third Millennium Medal (2000), the IEEE Signal Processing Society Meritorious Service Award (2001), and an IEEE Signal Processing Society Best Paper Award (2001). 